\title{
Chemokine receptor-like 2 is involved in ischemic brain injury
}

February 17, 2013 · Volume $6 \cdot$ Original Research

Robert M. Douglas ${ }^{1}$, Alice H. Chen ${ }^{1}$, Alejandra Iniguez ${ }^{1}$, Juan Wang ${ }^{1}$, Zhengxing Fu², Frank L. Powell Jr. ${ }^{2}$, Gabriel G. Haddad ${ }^{3}$, Hang Yao Ph.D. ${ }^{1}$

1 Department of Pediatrics, University of California-San Diego, La Jolla, CA, USA

2 Department of Physiology, University of California-San Diego, La Jolla, CA, USA

3 Department of Pediatrics, Neuroscience, University of California-San Diego, Rady Children's Hospital-San Diego, San Diego, CA, USA

Article citation: Douglas RM, Chen AH, Iniguez A, Wang J, Fu Z, Powell Jr. FL, Haddad GG, Yao H. Chemokine receptor-like 2 is involved in ischemic brain injury: CCRL2 is involved in ischemic brain injury. J Exp Stroke Transl Med 2013 Feb 17, Volume 6. pp 1-6. Online access at http://www.aestm.com/article/chemokine-receptor-like-2-is-involved-in-ischemic-brain-injury/

Correspondence should be sent to: Hang Yao, Ph.D. Department of Pediatrics University of California, San Diego, 9500 Gilman Drive, MC 0735, La Jolla, CA 92093-0735; Phone: 858-534-6970;Fax: 858-534-6972;E-mail: hyao@ucsd.edu.

\section{Abstract}

We examined the role of CCRL2 in ischemic brain injury using both in vitro and in vivo mouse stroke models. The expression of CCRL2 was enhanced at both the RNA and protein levels in cultured brain slices under ischemic conditions. Ischemia-induced cell death was reduced in brain slices derived from CCRL2 knockout (KO) mice in comparison with those from wild type (WT) mice. The infarct volume was smaller and neurological deficits were attenuated in CCRL2 KO mice when compared to WT mice subjected to a transient middle cerebral artery occlusion. Our data suggest that CCRL2 is involved in ischemia-induced brain injury in mice.

\section{Introduction}

Ischemic stroke not only results in necrosis at the infarct core but also triggers molecular changes in the surrounding peri-infarct tissues which can potentially lead to severe brain damage. Among the gene expression changes occurring in the peri-infarct region, chemokines and their receptors have drawn increasing attention since post-stroke inflammation plays a major role in the pathogenesis of stroke. Using an in vitro ischemia model that simulates penumbral cell death in mice, we screened about 46,000 mouse transcripts and found that chemokine (C-C motif) receptor-like 2 (CCRL2) was dramatically up-regulated in brain slices that were exposed to an ischemic solution mimicking the interstitial fluid found in the penumbra around the infarct core (Yao et al., 2009). In addition, recent studies have demonstrated that CCRL2 mRNA is increased in mouse brain subjected to a transient middle cerebral artery occlusion (tMCAO) (Brait et al., 2011), suggesting a potential involvement of CCRL2 in post-stroke pathology.

CCRL2 was first characterized in a mouse macrophage cell line more than a decade ago (Shimada et al., 1998). CCRL2 has significant homology to members of C-C chemokine receptor family. Members in this receptor family, such as CCR1, CCR2 and CCR5, have been implicated in cerebral ischemia (Offner et al., 2006, Reichel et al., 2006, Dimitrijevic et al., 2007). Studies in both human and animal models not only show a broad distribution profile of CCRL2 expression but also suggest a potential role of CCRL2 in the pathogenesis of several disease states. Available data demonstrate that CCRL2 mRNA is expressed in a variety of human tissues such as spleen, liver, lung, heart and brain (Fan et al., 1998, Migeotte et al., 2002). A similar distribution profile of CCRL2 expression has been observed in mice (Fan et al., 1998, Biber et al., 2003, Zuurman et al., 2003, Oostendorp et al., 2004). Studies in mice have suggested that CCRL2 may be involved in inflammatory lung disease and lgEmediated passive cutaneous anaphylaxis (Oostendorp et al., 2004, Zabel et al., 2008). In the CNS, enhanced expression of CCRL2 mRNA was observed in astrocytes, microglia and infiltrating macrophages in a murine model of multiple sclerosis (Brouwer et al., 2004) as well as in the cortex of LPS-treated mice (Zuurman et al., 2003).

In this work, we hypothesized that CCRL2 is involved in ischemia-induced brain injury in mice. We show that CCRL2 mRNA and protein are significantly increased in mouse brain slices exposed to an ischemic solution and that ischemia-induced cell death in brain slices derived from CCRL2 knockout (KO) mice was reduced compared with wild type (WT) slices. Furthermore, CCRL2 deletion in mice does not affect general physiological parameters but significantly alleviated brain damage and neurological deficits in mice subjected to tMCAO.

\section{Materials andMethods}

Procedures for organotypic brain slice cultures from the mouse brain. Cortical slice cultures were prepared and maintained as described previously (Kunkler and Kraig, 1997, Yao et al., 2007). Briefly, mice (6- to 8-day old) were anesthetized with halothane and the brains were removed and transferred into an ice-cold dissection medium (Gey's balanced salt solution supplemented with D-glucose $6.5 \mathrm{mg} / \mathrm{mL}$ ). Brain tissues were cut transversely into slices of $300 \mu \mathrm{m}$ each using a Vibratome ${ }^{\mathrm{TM}}$ 800-Mcllwain Tissue Chopper (Vibratome, St Louis, MO, USA). Slices were carefully transferred into $30 \mathrm{~mm}$ Millicell-CM tissue culture inserts (Millipore ${ }^{\mathrm{TM}}$, Bedford, MA, USA) and placed in $35 \mathrm{~mm}$ culture dishes (four to six slices per insert). Culture media contained basal Eagle medium (50\%), Earle's balanced salt solution (25\%), horse serum (25\%), and L-glutamine $(1 \mathrm{mM})$. The culture medium was supplemented with $50 \mathrm{U} / \mathrm{mL}$ penicillin/streptomycin and $36 \mathrm{mM}$ glucose. Cultures were maintained in a $5 \% \mathrm{CO}_{2}$, $37^{\circ} \mathrm{C}$ incubator for 14 days before experiments were performed. Culture medium was half-replaced on the second day after plating and twice a week thereafter until the day of treatment. Artificial cerebrospinal fluid (ACSF) was balanced with $5 \% \mathrm{CO}_{2}+$ room air. ACSF contained (in mM): $\mathrm{NaCl} 129, \mathrm{KCl} 5, \mathrm{CaCl}_{2} 1.3, \mathrm{MgCl}_{2} 1.5, \mathrm{NaHCO}_{3} 21$, glucose 10 (315 mOsm, pH 7.4). Ischemic solution (IS) was balanced with $15 \% \mathrm{CO}_{2}+$ $1.5 \% \mathrm{O}_{2}$. IS contained (in $\mathrm{mM}$ ): $\mathrm{NaCl} 47, \mathrm{KCl} 29$, $\mathrm{K}$-Gluconate $35, \mathrm{CaCl}_{2} 0.13, \mathrm{MgCl}_{2} 1.5, \mathrm{NaHCO}_{3} 4$, glucose 3 , glutamate 0.1 , Sucrose $65(315$ mOsm, pH 6.4).

Quantification of cell death. Cell damage was assessed with propidium iodide (PI) which is membrane impermeable and generally excluded from viable cells. However, if cell membranes are damaged, PI enters the cell and intercalates into the DNA with enhanced fluorescence. PI $(5 \mu \mathrm{g} / \mathrm{ml})$ 

was added into the culture medium 24 hours before any treatment and kept at the same concentration throughout the experiment PI fluorescence was observed on an inverted microscope equipped with a rhodamine filter set that has a 540-552nm band-pass filter for excitation and a 590 nm long-pass filter for emission (Zeiss Axiovert 200M microscope, Zeiss, Yena, Germany). A F-Fluar 5X objective (Zeiss, Yena, Germany) and the attached 12 bit CCD camera (C4742, Hamamatsu, Herrsching, Germany) were routinely used for image acquisition. The light source, microscope and the camera were controlled by a computer (Universal Imaging Corporation, Downington, PA). For data collection, the parameters of the microscope such as light intensity for excitation, exposure time and camera gain, were kept constant. Images were acquired and analyzed with MetaFluor imaging-processing software (Universal Imaging Corporation, Downington, PA).

Western blot. Tissues collected from cultured brain slices were transferred separately to lysis buffer: pH 7.5 [200 mM mannitol, 80 mM HEPES, 41 $\mathrm{mM} \mathrm{KOH}, 1$ tablet/50 $\mathrm{mL}$ of complete protease inhibitor tablets (Roche Diagnostics, Mannheim, Germany), and 230 IM phenylmethylsulfonyl fluoride, $\mathrm{pH}$ 7.5]. Sample protein concentration was determined using the BioRad DC Protein Assay Kit (BioRad, Hercules, CA, USA). Forty mg of total protein per sample were resolved on $10 \%$ precast NuPAGE Bis-Tris gels (Invitrogen, Carlsbad, CA, USA) and electrotransferred onto polyvinylidene difluoride membranes (Immobilin-P; Millipore). Membranes were then blocked in 5\% non-fat dry milk (Carnation, Nestle Food, Glendale, CA, USA) in phosphate-buffered saline (PBS)with $0.1 \%$ Tween 20 (Sigma, St Louis, MO) for $1 \mathrm{~h}$, and incubated with primary antibody overnight at $4^{\circ} \mathrm{C}$. Antibodies used for detecting CCRL2 expressionwere: rat anti-mouse monoclonal antibody (mAb) against CCRL2 (1:1000; antiCCRL2 mAb BZ5B8 was a kind gift of Dr. Brian Zabel, Stanford University School of Medicine) which was derived by immunizing rats with an amino-terminal mCCRL2 peptide corresponding to residues1-24 of mCCRL2 (Zabel et al., 2008). Other antibodies utilized were cleaved caspase 3 (Asp175; Cell Signaling; Boston, MA) and actin (Santa Cruz Biotechnology, Santa Cruz, CA). Membranes were then rinsed and incubated with secondary antibodies (peroxidase goat anti-rat (1:2000), peroxidase goat anti-rabbit $(1: 2000)$ and peroxidase rabbit anti-goat (1:2000), respectively (Cell Signaling) for $1.5 \mathrm{~h}$ at $23^{\circ} \mathrm{C}$, then rinsed, and stained with an enhanced chemiluminescence kit (Amersham, Chalfont, UK). Scanning densitometry of immunoblot films was performed on a Personal Densitometer SI scanner (Molecular Dynamics, Sunnyvale, CA, USA) and analyzed with the aid of ImageQuaNT image analysis software (Molecular Dynamics). Densitometry measurements of Western blots from each experimental group were obtained, and absolute values were normalized to actin.

Surgical procedures for middle cerebral artery occlusion (MCAO).Male WT and CCRL2 KO mice on a C57BL/6 background (Jackson Laboratory, Bar Harbor, ME) weighing 20-25 g at the time of surgery were used for all experiments. Mice were anesthetized with 1.5 to $2 \%$ isoflurane administered by mask and MCAO was performed as previously described (Kitagawa et al., 1998). Briefly, theright common carotid artery was exposed through a midline incisionin the neck and unilateral MCAO was performed by inserting a silicone rubber-coated monofilament (Doccol Corp., CA). The 6-0 silicone suture was introducedinto the common carotid artery and advanced into the internalcarotid artery for a distance of 10 to $11 \mathrm{~mm}$ from the commoncarotid artery bifurcation. In sham-operated mice, the carotid arteries were prepared surgically but the filament was not inserted. For reperfusion studies, the filament was left in place for 30 min for transient MCAO (tMCAO) and then withdrawn (for 24-hour reperfusion). This study was conducted in conformity with the Guiding Principles for Research Involving Animals and Human Beings and was approved by the UCSD Institutional Animal Care and Use Committee. Animal experiments were carried out in accordance with the National Institute of Health Guide for the Care and Use of Laboratory Animals (NIH Publications No. 80-23), revised 1996.

Physiological measurements. Mice were anesthetized with 1.5 to $2 \%$ isoflurane administered by mask and the right carotid artery was isolated for MCAO as described above. We catheterized the femoral artery (PE 10) for measuring mean arterial pressure (Gould P23 ID) and sampling blood for $\mathrm{pH}$ and blood gas analysis (Instrumentation Laboratory, GEM Premier 3000). Arterial samples (150 $\mu$ l) were taken anaerobically with a syringe before and 15 minutes after MCAO and analyzed immediately. Hematocrit was calculated by a blood gas analyzer.

Assessment of infarct volume.Animals were killed by an overdose of anesthesia at the time point of interest post-MCAO and the brain was removed and chilled at $-20^{\circ} \mathrm{C}$ for $3 \mathrm{~min}$ to slightly harden the tissue. One millimeter coronal sections were made from the olfactory bulb to the cerebellum using a brain matrix. Sections were incubated in $2 \% 2,3,5$-triphenyltetrazolium chloride(TTC) solution for 20 min at $37^{\circ} \mathrm{C}$. The sections were then rinsed with PBS and fixed with $4 \%$ paraformaldehyde for 30 min at room temperature. The infarct area, contralateral area and ipsilateral area in each individual brain sectionwas determined by using NIH Image 1.62 (Bethesda, MD, USA) and multiplied by the section thickness (1 mm) to get volume values. The volume values for each section were summed over the entire brain. To account for cerebraledema of the infarct, an indirect measurementof infarction was performed. Infarct volume was calculated as:[contralateral hemisphere volume-(ipsilateral hemispherevolume-measured infarct volume)] (Swanson et al., 1990).

Assessment of neurological deficits. Neurological evaluation of motor and sensory functions was carried out at two testing points: 20 min after MCAO and right before the designated time point when the mice were sacrificed for infarct volume measurement. The examiners were blinded as to the procedure that the mouse had undergone. Neurological deficits were determined using a 5-point scale system (Zhang et al., 1997). Briefly, each mouse was assigned a score of $0-4: 0=$ no observable neurological deficit; $1=$ forelimb weakness and torso turning to the ipsilateral side when held by tail; $2=$ circling to the affected side; $3=$ unable to bear weight on the affected side; and $4=$ no spontaneous locomotor activity or barrel rolling. Endurance in the wire-hanging test was measured on a horizontal steel wire $(1 \mathrm{~mm})$ stretched horizontally $50 \mathrm{~cm}$ above a foam padand the experimental animal was suspended by its forelimbs on the wire. The time until the animal fell was recorded which was used as a measure of grasping ability and forelimb strength (Hattori et al., 2000).

Statistical Analyses. Values are presented as means \pm standard deviations (SD). Differences in main effects between two groups were examined with a student $t$-test. Differences among multiple groups were determined by a two-way ANOVA followed by Bonferroni's post test, using GraphPad Prizm software (GraphPad, San Diego CA). The criterion for statistical significance was a $p$ value of $<0.05$.

\section{Results}

\section{Ischemia up-regulated the expression of CCRL2 in cultured brain slices.}

The expression profile of CCRL2 mRNA was studied in WT brain slices incubated with artificial cerebral spinal fluid (ACSF; control) or with ischemic solution (IS) for 2, 6 and 12 hours. Fig. 1A shows the IS-induced mean fold changes of CCRL2 mRNA at each time point (relative to the CCRL2 expression of ACSF-treated brain slices, $n=3, p<0.05$ when compared to ACSF group). CCRL2 mRNA was increased about ten fold after 2 hours of IS treatment. This increased expression continued at 6 hours and further increased to 25 fold at 12 hours of IS treatment, suggesting that the increased expression of CCRL2 is associated with prolonged ischemia. To determine if the alteration seen at the RNA level was reflected at the protein level, Western blot analysis was conducted at 14 hours following IS treatment. As shown in Fig. 1B, CCRL2 protein was increased in the IS-treated slices. An increased cleaved caspase-3 was also detected in these slices, suggesting that cells were undergoing apoptotic cell death in IS-treated slices. Fig. 1C \& D show the statistical analysis of the changes in CCRL2 and cleaved-caspase-3 protein after 14 hours of ischemia in brain slices. Compared with the ACSF control group, both CCRL2 and C-Caspase-3 were significantly increased in the brain slices following 14 hours of IS treatment ( $n=3$ for each group, $\left.{ }^{*}: p<0.05\right)$. 

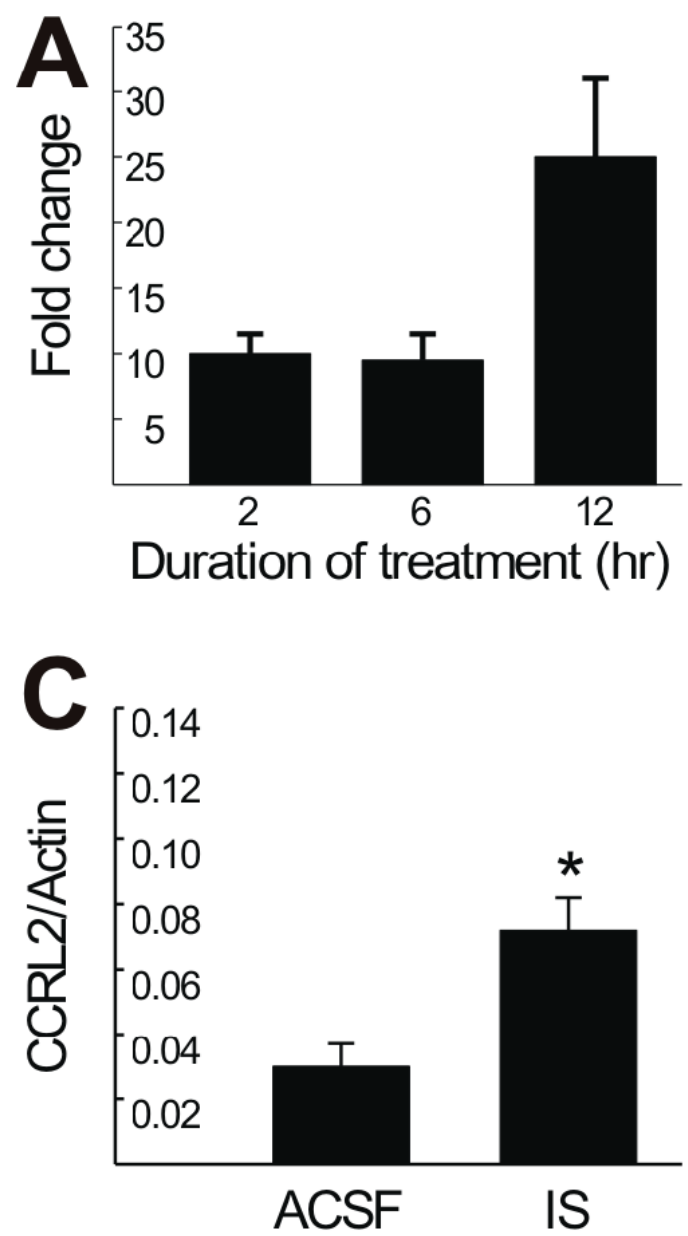

B

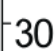

30

25

20

15

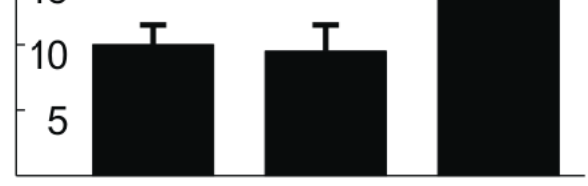

2

6

12

Duration of treatment (hr)
IS ACSF

\section{CCRL2}

C-Casp 3

$\beta$-actin
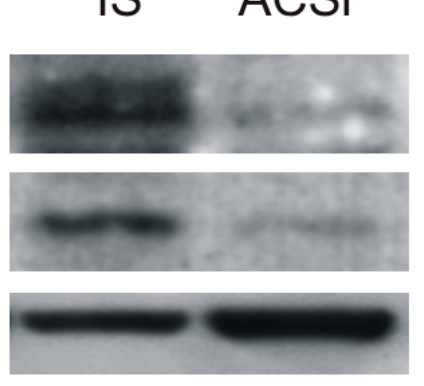

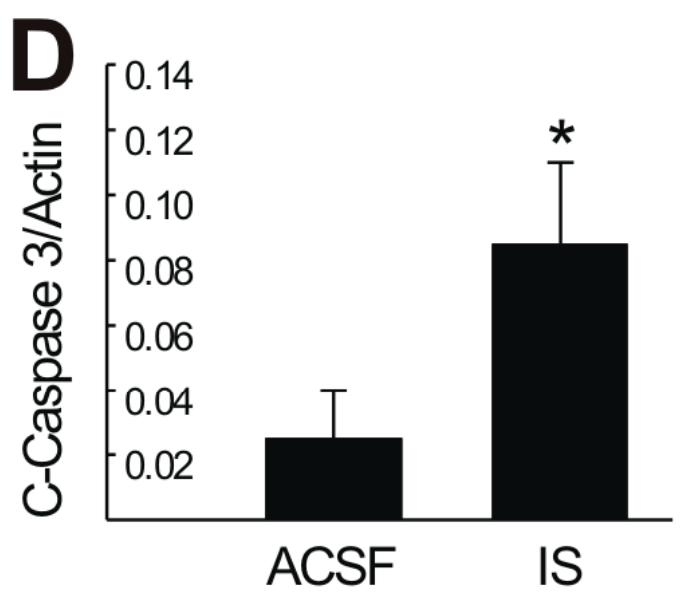

Figure 1.

CCRL2 is expressed in cultured mouse brain slices and up-regulated following simulated ischemia. A, CCRL2 mRNA was upregulated in brain slice cultures following ischemia in WT mice. Bar graph showing the fold change of CCRL2 mRNA in IS group over ACSF control group at each time point. B, Representative Western blot analysis of CCRL2 and cleaved-caspase-3 in brain slices after 14 hours of ischemia treatment. C-D, Quantification of the Western blot analysis of CCRL2 and cleaved-caspase-3 in ischemia-treated brain slices (*: $p<0.05)$. Values in $A, C$ and $D$ are mean $\pm S D$ of triplicate analyses on samples pooled from 48 brain slices per experimental group.

CCRL2 deletion reduced ischemia-induced cell death in cultured brain slices.

Cortical slices derived from both CCRL2 KO and WT mice were treated with IS. Cell death was assessed by propidium iodide (PI) fluorescence (PI uptake) in brain slices following the IS treatments. As shown in Fig. 2A, in both WT and CCRL2 KO slices, exposure to the IS solution caused PI uptake in injured cells and PI uptake increases with prolonged IS exposure duration. However, the time-lapsed increase of PI uptake in CCRL2 KO slices was at a significantly slower pace. For example, at 24 and 48 hours following IS treatment, the mean PI uptake in CCRL2 KO slices is significantly less than that in WT slices (Fig. 2B, $n=18$ for each group, $p<0.05$ ).

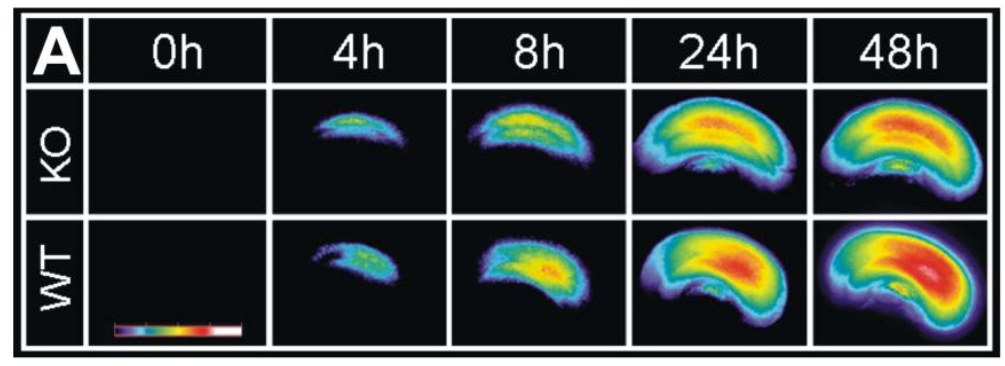

Figure 2.

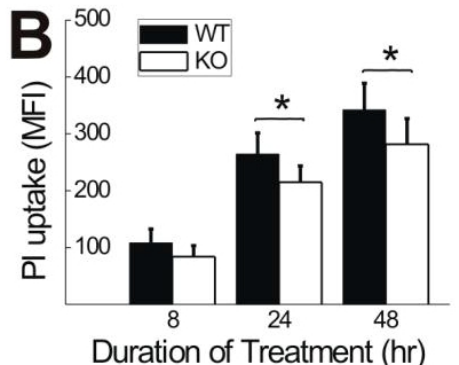

Duration of Treatment (hr)

Ischemic cell death is reduced in cultured cortical brain slices derived from CCRL2 KO mice. A, Pseudocolor images of PI 
Robert M. Douglas CCRL2 is involved in ischemic brain injury

J Exp Stroke Transl Med (www.aestm.com) Vol 6 pp $4-6$ fluorescence in cortical brain slices acquired at the indicated time points. The pseudocolor scale is shown on the left bottom, with purple and white indicating the least and the most intense levels of $\mathrm{PI}$ fluorescence, respectively. B, bar graph showing the comparison of the mean PI uptake between WT and CCRL2 KO brain slices. MFI=mean fluorescent intensity. *: $p<0.05$.

Physiological parameters in CCRL2 KO and WT mice following MCAO.

Arterial blood gases and pressure were measured in both WT and CCRL2 KO mice before and 15 min after MCAO surgery ( $\mathrm{n}=6$ both groups). As shown in Table 1, there were no significant differences between WT and CCRL2 KO mice for each variable before or during MCAO.

\begin{tabular}{|c|c|c|c|c|c|c|}
\hline \multicolumn{7}{|c|}{ Physiologic variables in CCRL2 KO and WT control mice before and 15 min after MCAO } \\
\hline & Sampling & pH & $\mathrm{pO}_{2}$ & $\mathrm{pCO}_{2}$ & Hct & MAP \\
\hline \multirow{2}{*}{ WT } & Before & $7.40 \pm 0.01$ & $162.67 \pm 3.33$ & $35.17 \pm 1.11$ & $40.17 \pm 0.40$ & $86.00 \pm 2.31$ \\
\hline & During & $7.37 \pm 0.03$ & $143.17 \pm 8.01$ & $41.00 \pm 3.18$ & $38.67 \pm 0.84$ & $82.67 \pm 2.38$ \\
\hline \multirow{2}{*}{$\mathrm{KO}$} & Before & $7.41 \pm 0.01$ & $163.33 \pm 2.88$ & $28.17 \pm 1.89$ & $40.40 \pm 0.89$ & $80.83 \pm 3.96$ \\
\hline & During & $7.30 \pm 0.05$ & $155.33 \pm 6.34$ & $41.50 \pm 3.60$ & $39.17 \pm 1.35$ & $82.50 \pm 1.18$ \\
\hline
\end{tabular}

Table 1.

Physiological parameters: arterial $\mathrm{pH}$, oxygen partial pressure ( $\mathrm{pO} 2$ ) and $\mathrm{CO} 2$ partial pressure (pCO2), and mean arterial pressure measured before and after MCAO. Animals were weighed before onset of the experiment. No statistical significant differences were found between groups ( $n=$ six animals per group).

\section{Brain lesion sizes in CCRL2 KO and WT mice subjected to MCAO.}

Both permanent MCAO (pMCAO) and transient MCAO (tMCAO) mouse models were used to study the effect of CCRL2 deletion on the ischemic brain injury. In mice subjected to a $24 \mathrm{~h}$ pMCAO, the mean infarct volume in CCRL2 KO ( $\mathrm{n}=8$ ) mice was not significantly different from that in WT mice (Fig. 3B, $n=9, p>0.05)$. However, in mice subjected to a tMCAO, the mean infarct volume in CCRL2 KO mice $\left(6.66 \pm 4.41 \mathrm{~mm}{ }^{3}\right.$, $\left.\mathrm{n}=9\right)$ was significantly smaller than that in WT mice $\left(50.04 \pm 9.26 \mathrm{~mm}^{3}, \mathrm{n}=10, \mathrm{p}<0.001\right.$, Fig. 3A, B).
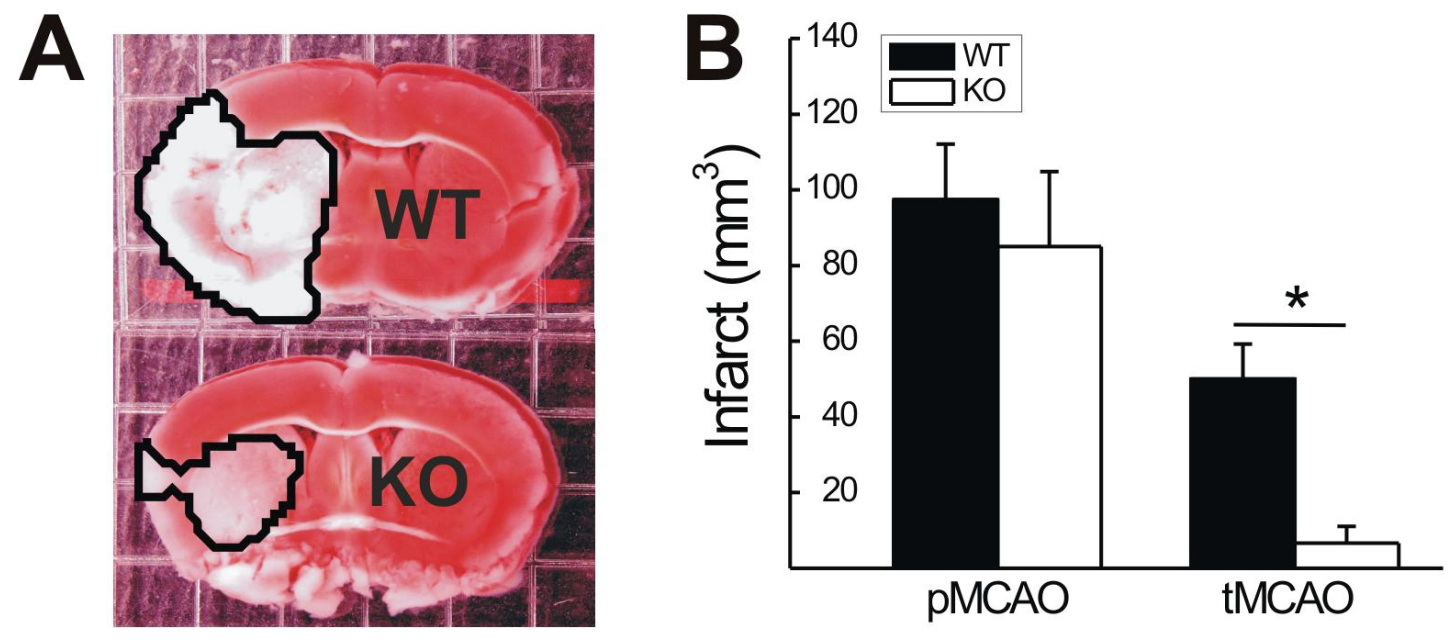

\section{Figure 3.}

CCRL2 gene deletion reduced the brain lesion in mice subjected to a tMCAO. A, TTC-stained brain sections obtained from WT (top) and CCRL2 KO mice (bottom) subjected to a tMCAO. Note that the infarct area (in white) is much smaller in CCRL2 KO than in WT mice. B, bar graph showing the comparison of the mean infarct volume between WT and CCRL2 KO mice subjected to $\mathrm{pMCAO}$ or $\mathrm{MCAO}$. Values are mean \pm SD of analyses on samples pooled from 8-10 animals per experimental group. *: $\mathrm{p}<0.05$.

\section{Neurobehavioral assessment in CCRL2 KO and WT mice subjected to MCAO.}

Neurological score assessment and wire-hanging test were conducted for each mouse subjected to pMCAO or tMCAO before the animals were sacrificed for TTC staining. Fig. 4A shows the comparison of neurological scores between CCRL2 KO and WT mice. In pMCAO groups, the neurological scores in CCRL2 KO ( $n=8)$ mice were not significantly different from that in WT mice $(n=9, p>0.05)$. In the tMCAO group, however, the neurological scores were significantly lower in CCRL2 KO $(1.4 \pm 0.2, n=9)$ than that in WT mice $(2.9 \pm 0.4, n=10, p<0.05)$, suggesting a significantly faster functional recovery in CCRL2 KO than in WT mice (Fig. 4A).

In the tMCAO group, a wire-hanging test was also performed following the neurological score assessment. As shown in Fig. 4B, latencies to fall are significantly longer in CCRL2 KO mice $(n=9)$ than that in WT mice $(n=10, p<0.05)$, suggesting a better functional recovery from MCAO in CCRL2 $\mathrm{KO}$ mice than in WT mice. 


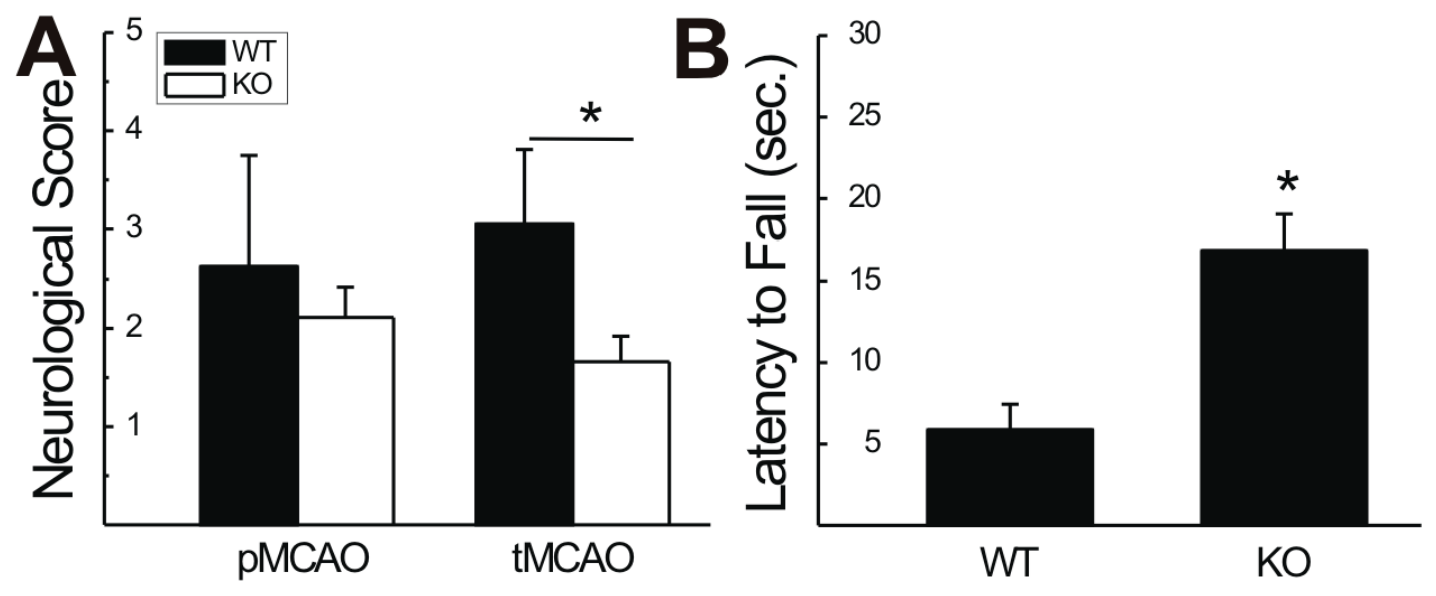

Figure 4.

Neurobehavioral assessment in WT and CCRL2 KO mice subjected to MCAO. A, bar graph showing mean neurological scores assessed in mice subjected to pMCAO or tMCAO right before being sacrificed at $24 \mathrm{~h}$ for infarct volume measurement. B, bar graph showing mean latency to fall in tMCAO mice right before being sacrificed at $24 \mathrm{~h}$ for infarct volume assessment. Values are mean \pm SD pooled from 8-10 animals in each experimental group.

\section{Discussion}

In this study, we show that the expression of CCRL2, a chemokine receptor, is up-regulated in mouse brain slices under ischemic conditions. The deletion of CCRL2 not only reduced the ischemia-induced cell death in brain slices but attenuated tMCAO-induced brain injury and neurological deficits in mice. Therefore, CCRL2 is involved in brain injury in the mouse stroke model.

Previous studies have shown that CCRL2 mRNA is expressed in mouse brain and particularly in glial cells under pathological conditions (Zuurman et al., 2003). In a transient MCAO model (30 min MCAO + reperfusion), a significant up-regulation of CCRL2 mRNA in the mouse brain was observed after a $24 \mathrm{~h}$ - or 72 h-reperfusion (Brait et al., 2011). In our previous study, we used microarray analysis to show that CCRL2 mRNA was upregulated in brain slices treated with ischemia solution (Yao et al., 2009). The current work has further confirmed that CCRL2 mRNA and protein were increased by $14 \mathrm{~h}$ of ischemia. Our data also show an increase in cleaved caspase 3 which is suggestive of apoptotic cell death and increased PI uptake which indicates both apoptotic and necrotic cell death at a later time point. The early increase in CCRL2 expression may indicate that CCRL2 initiates an inflammatory cascade that induces both apoptosis and necrosis. Indeed, chemokine receptors in the C-C chemokine receptor family, such as CCR1, CCR2 and CCR5, have been implicated in CNS disorders (Offner et al., 2006, Reichel et al., 2006, Dimitrijevic et al., 2007). Although we have not analyzed the function of CCRL2 in the current study, it is tempting to believe that the CCRL2 could be a new target that contributes to CNS physiology and pathology.

Our results show that mice lacking CCRL2 had major decreases in infarct volume and showed significant improvement in neurological outcomes in tMCAO+24 h-reperfusion group. The mechanism underlying the protective effect of CCRL2 deletion is not immediately clear. Available data and our observations suggest that there is no abnormal phenotype found in the CCRL2 KO mice (Zabel et al., 2008). Arterial blood gases and pressure were not significantly different between CCRL2 KO and WT before or after MCAO. This supports the idea that the differences in the response to MCAO with CCRL2 deletion are not the result of differences in respiratory or cardiovascular function prior to ischemia. However, there was a nonsignificant trend for lower arterial $\mathrm{P}_{\mathrm{CO} 2}$ in CCRL2 KO mice before MCAO (Table 1), suggesting hyperventilation. Further experiments are necessary to determine if CCRL2 normally plays a role in control of breathing by recruiting proinflammatory signals to respiratory centers in the brain since inflammatory signals have been recognized recently as important modulators of cardiovascular and respiratory control (Powell and Kou, 2011, Waki et al., 2011).

The potential alteration of cerebral vascular structure in the CCRL2 KO mice could be a factor that needs further investigation since gene knockout in mice could change the cerebral vascular anatomy as documented previously (Yang et al., 2007). As such, it is possible that the neurovascular territory of the middle cerebral artery in CCRL2 KO mice could have been changed as compared to WT controls and which, as a result, could have led to reduced infarct sizes. Nevertheless, we believe it is less likely a major factor contributing to the reduction of brain injury in this study since the attenuation of brain injury only occurred in the $\mathrm{tMCAO}+24 \mathrm{~h}$-reperfusion group but not in the $24 \mathrm{~h}$-pMCAO group. This suggests that an increase in CCRL2 expression may have a detrimental effect on brain injury at cellular and molecular levels.

Our IS model was developed to mimic penumbral cell death in ischemic stroke (Yao et al., 2007). The significant reduction of IS-induced cell death in the in vitro CCRL2 KO brain slices indicates that a profound protection of penumbral cells should occur in the in vivo penumbral area. Along the same line, the larger the penumbral region that exists, the more protection will be demonstrated in CCRL2 KO mice subjected to MCAO. Indeed, after the deletion of CCRL2 gene, our in vivo tMCAO mice showed more pronounced brain protection than pMCAO mice which may be indicative of the prior having a larger penumbral region than the latter. This is in agreement with the well known fact that pMCAO produces a more severe and rapid brain infarction with a smaller and more short-lived penumbra than is seen with $\mathrm{MCAO}$, in which, infarcts are surrounded by a large ischemic penumbra(Lo et al., 2003). Thus, the neuroprotective effect of CCRL2 deletion found in the in vitro brain slices and in vivo stroke model may suggest that CCRL2 plays a role in ischemia-induced cell death.

Taken together, our study suggests that CCRL2 is an important component of ischemia/reperfusion brain injury. As a C-C chemokine receptor, CCRL2 may not only mediate proinflammatory processes in the lesion area but also participate in the direct modulation of cell death following ischemia. Therefore, CCRL2 is a potential therapeutic target for stroke treatment.

\section{References}



CCL7, and CCL8. J Leukoc Biol 74:243-251.

Brait VH, Rivera J, Broughton BR, Lee S, Drummond GR, Sobey CG (2011) Chemokine-related gene expression in the brain following ischemic stroke: no role for CXCR2 in outcome. Brain research 1372:169-179.

Brouwer N, Zuurman MW, Wei T, Ransohoff RM, Boddeke HW, Biber K (2004) Induction of glial L-CCR mRNA expression in spinal cord and brain in experimental autoimmune encephalomyelitis. Glia 46:84-94.

Dimitrijevic OB, Stamatovic SM, Keep RF, Andjelkovic AV (2007) Absence of the chemokine receptor CCR2 protects against cerebral ischemia/reperfusion injury in mice. Stroke 38:1345-1353.

Fan P, Kyaw H, Su K, Zeng Z, Augustus M, Carter KC, Li Y (1998) Cloning and characterization of a novel human chemokine receptor. Biochem Biophys Res Commun 243:264-268.

Hattori K, Lee H, Hurn PD, Crain BJ, Traystman RJ, DeVries AC (2000) Cognitive deficits after focal cerebral ischemia in mice. Stroke; a journal of cerebral circulation 31:1939-1944

Kitagawa K, Matsumoto M, Yang G, Mabuchi T, Yagita Y, Hori M, Yanagihara T (1998) Cerebral ischemia after bilateral carotid artery occlusion and intraluminal suture occlusion in mice: evaluation of the patency of the posterior communicating artery. J Cereb Blood Flow Metab 18:570-579.

Kunkler PE, Kraig RP (1997) Reactive astrocytosis from excitotoxic injury in hippocampal organ culture parallels that seen in vivo. J Cereb Blood Flow Metab 17:26-43.

Lo EH, Dalkara T, Moskowitz MA (2003) Mechanisms, challenges and opportunities in stroke. Nat Rev Neurosci 4:399-415.

Migeotte I, Franssen JD, Goriely S, Willems F, Parmentier M (2002) Distribution and regulation of expression of the putative human chemokine receptor HCR in leukocyte populations. Eur J Immunol 32:494-501.

Offner H, Subramanian S, Parker SM, Afentoulis ME, Vandenbark AA, Hurn PD (2006) Experimental stroke induces massive, rapid activation of the peripheral immune system. J Cereb Blood Flow Metab 26:654-665.

Oostendorp J, Hylkema MN, Luinge M, Geerlings M, Meurs H, Timens W, Zaagsma J, Postma DS, Boddeke HW, Biber K (2004) Localization and enhanced mRNA expression of the orphan chemokine receptor L-CCR in the lung in a murine model of ovalbumin-induced airway inflammation. $J$ Histochem Cytochem 52:401-410.

Powell FL, Kou YR (2011) Inflammation and cardio-respiratory control. Foreword. Respir Physiol Neurobiol 178:359-361.

Reichel CA, Khandoga A, Anders HJ, Schlondorff D, Luckow B, Krombach F (2006) Chemokine receptors Ccr1, Ccr2, and Ccr5 mediate neutrophil migration to postischemic tissue. J Leukoc Biol 79:114-122.

Shimada T, Matsumoto M, Tatsumi Y, Kanamaru A, Akira S (1998) A novel lipopolysaccharide inducible C-C chemokine receptor related gene in murine macrophages. FEBS Lett 425:490-494.

Swanson RA, Shiraishi K, Morton MT, Sharp FR (1990) Methionine sulfoximine reduces cortical infarct size in rats after middle cerebral artery occlusion. Stroke 21:322-327.

Waki H, Gouraud SS, Maeda M, Raizada MK, Paton JF (2011) Contributions of vascular inflammation in the brainstem for neurogenic hypertension. Respir Physiol Neurobiol 178:422-428.

Yang LV, Radu CG, Roy M, Lee S, McLaughlin J, Teitell MA, Iruela-Arispe ML, Witte ON (2007) Vascular abnormalities in mice deficient for the G protein-coupled receptor GPR4 that functions as a pH sensor. Molecular and cellular biology 27:1334-1347.

Yao H, Felfly H, Wang J, Zhou D, Haddad GG (2009) DIDS protects against neuronal injury by blocking Toll-like receptor 2 activated-mechanisms. J Neurochem 108:835-846

Yao H, Shu Y, Wang J, Brinkman BC, Haddad GG (2007) Factors influencing cell fate in the infarct rim. J Neurochem 100:1224-1233.

Zabel BA, Nakae S, Zuniga L, Kim JY, Ohyama T, Alt C, Pan J, Suto H, Soler D, Allen SJ, Handel TM, Song CH, Galli SJ, Butcher EC (2008) Mast cell-expressed orphan receptor CCRL2 binds chemerin and is required for optimal induction of lgE-mediated passive cutaneous anaphylaxis. $J$ Exp Med 205:2207-2220

Zhang RL, Chopp M, Zhang ZG, Jiang Q, Ewing JR (1997) A rat model of focal embolic cerebral ischemia. Brain Res 766:83-92.

Zuurman MW, Heeroma J, Brouwer N, Boddeke HW, Biber K (2003) LPS-induced expression of a novel chemokine receptor (L-CCR) in mouse glial cells in vitro and in vivo. Glia 41:327-336.

\section{Funding Statement}

This project was supported by NIH PO1 HD 32573, NIH RO1 HL 66237.

\section{Acknowledgements}

We thank Dr. Brian A. Zabel from Department of Pathology, Stanford University for providing CCRL2 antibody. We thank UCSD Neuroscience Microscopy Shared Facility (NINDS P30 NS047101) for providing the imaging support. 